\title{
Effects of Heat Exchange Tube Structural Parameters on Performance of Vehicle Radiator
}

\author{
Zeyan Liu, Xilei Kong, Xiaobiao Han, Hui Liu, Quanzhen Wang, Zhenling Wang \\ School of Transportation and Vehicle Engineering, Shandong University of Technology, Zibo, China \\ Email:sdutliu@163.com
}

How to cite this paper: Liu, Z.Y., Kong, X.L., Han, X.B., Liu, H., Wang, Q.Z. and Wang, Z.L. (2020) Effects of Heat Exchange Tube Structural Parameters on Performance of Vehicle Radiator. Journal of Power and Energy Engineering, 8, 1-7.

https://doi.org/10.4236/jpee.2020.89001

Received: July 30,2020

Accepted: September 5, 2020

Published: September 8, 2020

Copyright $\odot 2020$ by author(s) and Scientific Research Publishing Inc. This work is licensed under the Creative Commons Attribution International License (CC BY 4.0).

http://creativecommons.org/licenses/by/4.0/

\begin{abstract}
In order to improve the performance of vehicle radiators, a two-dimensional heat transfer steady-state model of the radiator was set up. The influence of the structural parameters (axial ratio) of the heat exchange tube on the windward side on the heat transfer performance of the radiator was studied. With the increase of the axial ratio of the heat exchange tube on the windward side, the heat exchange capacity of the heat exchange tube surface slightly decreases. The heat exchange area increases significantly, which increases the total heat exchange of the radiator and improves the heat transfer performance of the radiator. When the axial ratio increases from 1.0 to 2.0, the average surface heat transfer capacity decreases from $5664.16 \mathrm{~W} / \mathrm{m}^{2}$ to $5623.57 \mathrm{~W} / \mathrm{m}^{2}$.
\end{abstract}

\section{Keywords}

Vehicle Radiator, Heat Transfer Performance, Heat Exchange Tube Structure Parameters, Axial Ratio

\section{Introduction}

With the diversified development of the automobile industry, the power of automobile engines is getting larger and larger, and the thermal load of engines is gradually increasing. During engine operation, the highest combustion temperature can reach more than $2800 \mathrm{~K}$; the average temperature of the combustion chamber can also reach more than $1300 \mathrm{~K}$ when the engine is idling or medium speed. As the core component of the automobile cooling system, the radiator undertakes the heat dissipation work of the entire engine, and the heat energy generated by the engine cannot be converted into mechanical energy is released in time so that the engine is maintained at a relatively stable and appropriate operating temperature range. The optimization will definitely promote the over- 
all optimization and upgrading of the cooling system, provide strong support for the improvement of the performance of the vehicle, and improve the ability of the car to work under complex working conditions [1].

In terms of the optimization of the radiator structure parameters, Wang Yanmei [2] simulated the heat transfer process of the assembled radiator heat exchange tubes and fins, obtained the structural parameters with the best heat dissipation performance, and the correctness of the numerical simulation results is verified through wind tunnel experiments. Máté Petrik [3] studied the performance parameters of cross-flow gas-liquid fins heat exchanger. The results show that the main parameters that affect the performance of the radiator are the radiator width and the fin parameters. Zheng Mingqiang [4] conducted a combination of simulation and optimization on the parameters such as the opening angle, spacing and thickness of the radiator shutters, which effectively improved the heat transfer coefficient, pressure drop at the inlet and outlet, and the comprehensive heat transfer performance. This provides a design reference for the optimization of the intercooler. Moon M A [5] calculated the heat transfer and friction loss in a rectangular channel with pin fins, and compared it with the heat transfer and friction loss of a circular pin fin. Compared with the reference pin fin, the fan pin fin has improved heat transfer and pressure drop. Pan An [6] established a three-dimensional model of the local fins and the overall radiator of the tube-belt radiator, and carried out the fin structure of the radiator. The results show that after the fin structure is optimized, the air side pressure drop is basically unchanged, but the heat dissipation increased by $11.6 \%$. Shi Miao [7] took straight finned heat pipe radiators as the research object, studied the influence of fin thickness, spacing, height, width and heat pipe diameter on the heat transfer performance of fins, and obtained the structural optimization scheme of the radiator, analyzed the main factor of influence, and has certain guiding significance for engineering application.

In summary, most scholars have focused on the influence of radiator shutter thickness, spacing, height, width, and angle on the effects of the heat dissipation system. However, there is no systematic study on the influence of the structure parameters of a single heat exchange tube on the heat dissipation performance. Therefore, a single heat exchange tube is set as the research object, the influence law of the long axis ratio and short axis ratio on the windward side for the heat transfer performance of the radiator was analyzed. The theoretical support for the improvement of the heat exchange structure was provided.

\section{Model Building}

The heat exchange tube is made of aluminum with a wall thickness of $0.5 \mathrm{~mm}$, the gap between the heat exchange tubes is $3 \mathrm{~mm}$, and the two-dimensional width of the fluid in the tube is $1.5 \mathrm{~mm}$. In order to allow sufficient heat exchange between the outside medium and the aluminum structure, the space on the windward side of the cooling fluid is $5 \mathrm{~mm}$, and the space on the rear side of 
the cooling fluid is $10 \mathrm{~mm}$ [8]. The simplified heat exchange tube is an axisymmetric structure, so a half model of the heat exchange tube is established when building the physical model. The basic structure is shown in Figure 1. Unstructured mesh is used for model discretization. The number of meshes is 18,795 . The cooling process of a single heat exchange tube can be expressed as a flow-heat transfer process. For the mathematical description equations of the model, please refer to [4] and [8].

The initial conditions and boundary conditions of the calculation model are shown in Table 1. In this paper, the axial ratio of the core structure parameters on the windward side of the heat exchange tube is selected as the changing parameter (Figure 2), and the research scope is selected from 1.0, 1.2, 1.4, 1.6, 1.8, and 2.0.

\section{Results and Analysis}

Figure 2 shows the effect of the change of the long and short axis ratio on the surface pressure of the heat exchange tube (take $\mathrm{X}$ to represent the distance from the heat exchange tube to the inlet at different positions, unit: $\mathrm{m}$, the same below). It can be seen from Figure 3, when the air flows through the top of the long diameter $\mathrm{X}=0.005$, its pressure drops suddenly due to it is blocked by the front of the heat exchange tube. When the airflow reaches $X=0.01$, the overall impact is minimal, and the shape of the windward side has an impact on the air the effect of flow basically disappears here. With the flow of air on the surface, the air is affected by the heating effect of the heat exchange tube. The pressure begins to show a linear change and finally reaches the peak. After reaching the rear of the heat exchange tube, the surface pressure begins to rise under the interaction of the air circulation area suddenly becoming larger and the heat

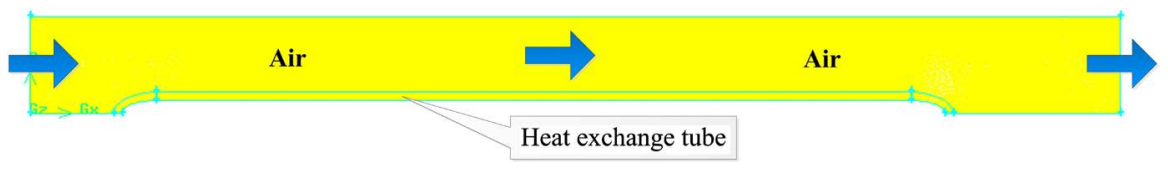

Figure 1. Physical model.

Table 1. Initial and boundary conditions.

\begin{tabular}{ccc}
\hline Serial number & Parameter name & Parameter value \\
\hline 1 & Inlet flow rate & $5 \mathrm{~m} / \mathrm{s}$ \\
2 & Inlet temperature & $298 \mathrm{~K}$ \\
3 & strength & $4 \%$ \\
4 & Entrance size & $5.75 \mathrm{~mm}$ \\
5 & Wall thickness & $0.5 \mathrm{~mm}$ \\
6 & Inner wall temperature & $353 \mathrm{~K}$ \\
7 & Thermal Conductivity & $\lambda=7.174+2.704 \times 10^{-3} T$ \\
8 & Specific heat capacity & $C_{P}=3447.584-24.451 T+0.079 T^{2}$ \\
\hline
\end{tabular}




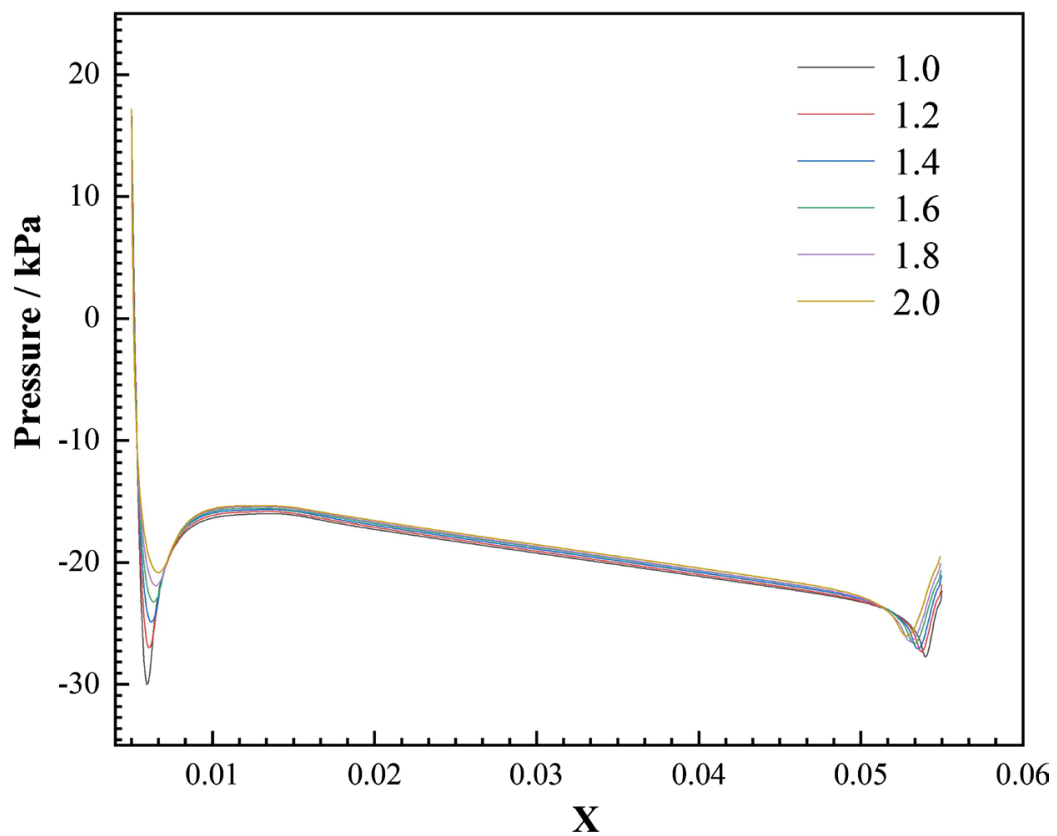

Figure 2. Effects of the change of the long and short axis ratio on the surface pressure of the heat exchange tube.

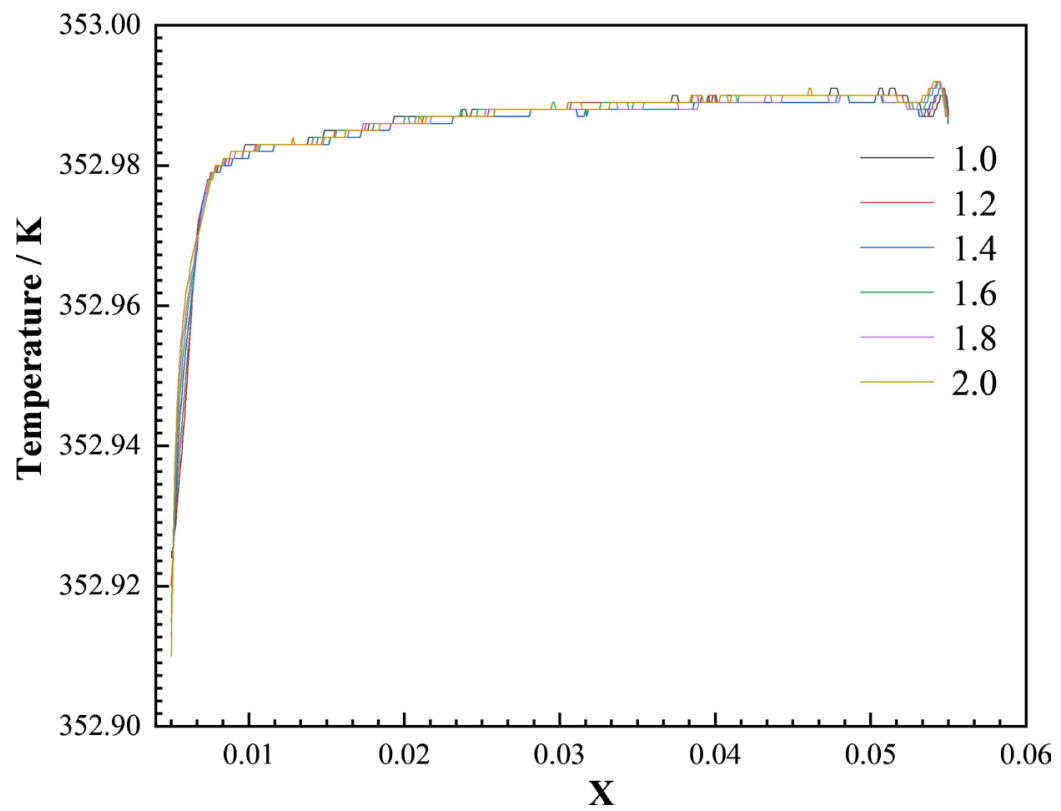

Figure 3. Effects of the change of the long and short axis ratio on the surface temperature of the heat exchange tube.

exchange tube heating the air. With the increase of the ratio of the long diameter and the short diameter of the heat exchange tube, the pressure at the junction of the heat exchange tube and the air is greatly increased, and the change of the structure on the windward side reduces the erosion strength of the heat exchange tube caused by the incoming airflow, flow variation at different locations at the junction results in pressure variation.

Figure 3 shows the effect of the change of the long and short axis ratio on the 
surface temperature of the heat exchange tube. The temperature of the inner wall surface of the heat exchange tube is $353 \mathrm{~K}$, which can be seen from Figure 4, the temperature at the air inlet changes the most, the air impacts the windward side, and the airflow intensity is high. So the airflow has the best cooling effect outside the heat exchange tube. With the change of the structure of the heat exchange tube, before reaching the linear structure, the intensity of the airflow changes as well, and the cooling capacity of the radiator tube evidently drops, which makes the upper temperature of the junction rise. On the horizontal boundary, the heat dissipation effect is mainly affected by the temperature of the flowing air, but the air continuously exchanges heat with the heat exchange tube during the flow process, causing the temperature to raise continuously, hence the cooling effect on the heat exchange tube becomes poor, and the temperature at the junction rises slowly. On the leeward side of the heat exchange tube, the air that has been heated to a higher temperature, the larger the circulation range, and the backflow of air on the rear side cause the surface temperature to fluctuate.

Figure 4 shows the effect of the change in the ratio of the long and short axis on the heat exchange on the surface of the heat exchange tube. It can be seen from Figure 4 that at the entrance under the influence of the combined effect of air temperature and airflow flow path changes, the heat dissipation effect is the best, and the heat exchange amount is the most at this time. When flowing through the horizontal surface of the heat exchange tube, the air flow on the upper side of the heat exchange tube tends to be stable. The heat exchange amount is mainly affected by the air temperature $\Delta \mathrm{T}$. The variation of heat dissipation shows a stable curve. The change in the leeward side shape makes the high-speed flowing air and the tail of the heat exchange tube fail to make sufficient contact,

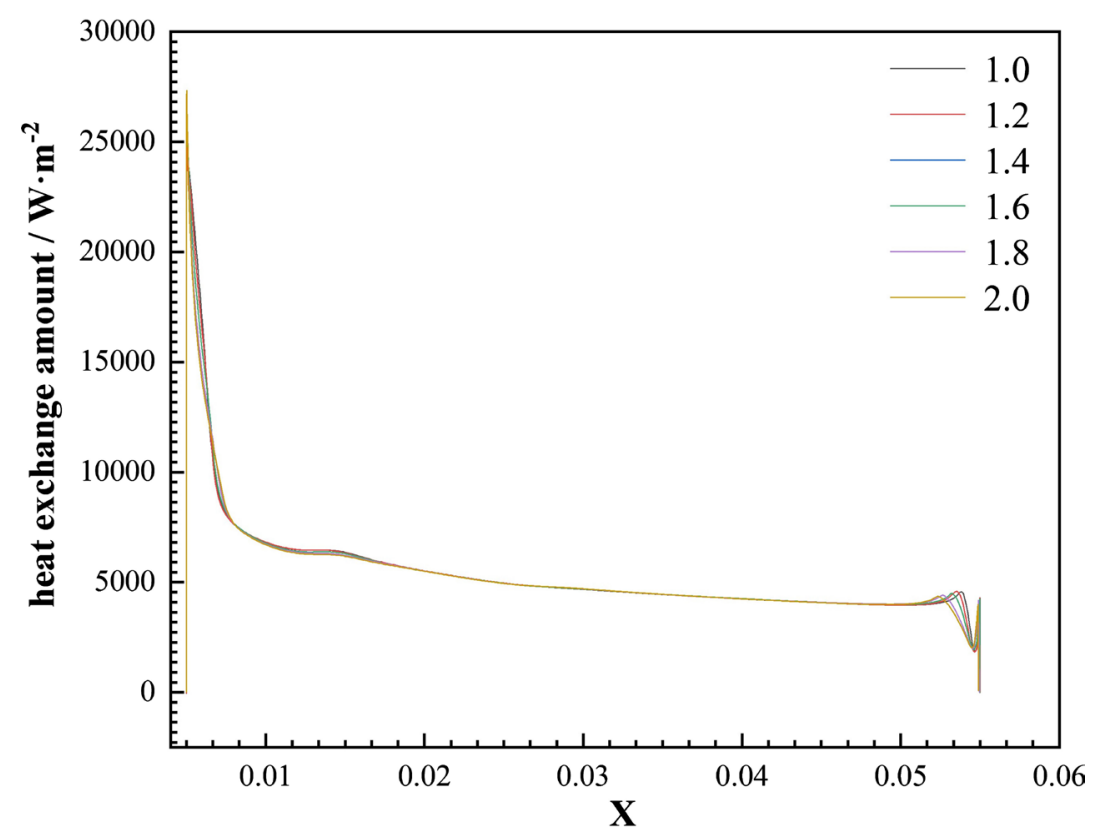

Figure 4. Effects of the change of the long and short axis ratio on the heat exchange on the surface of the heat exchange tube. 
and the heat exchange amount on the surface of the heat exchange tube decreases sharply. As the ratio of the long diameter and the short diameter increases, the structural resistance of the air at the inlet decreases, and the heat exchange amount on the windward side decreases significantly; on the rear side of the heat exchange tube, the ratio of the long diameter to the short diameter of the heat exchange tube increases, the surface temperature of the heat exchange tube and the temperature difference at the junction become larger, meanwhile the heat exchange amount begins to increase.

Figure 5 shows the effects of the change of the long and short axis ratio on the heat transfer capacity of the heat exchange tube surface. It can be seen from Figure 5, the average heat transfer capacity on the surface of the heat exchange tube decreases slightly as the axial ratio increases. For the reason is that with the increase of the long and short axis ratio, the degree of sudden variation in the windward surface structure of the heat exchange tube decreases, and the degree of change to the air streamline reduces, thereby reducing the chance of collision between gas molecules and the heat exchange wall, so that the intensity of heat exchange is slightly reduced. However, due to the increase of axial ratio, the heat exchange area on the windward side of the heat exchange tube is obviously increased, thereby increasing the overall heat exchange of the radiator and improving the performance of the radiator.

\section{Conclusion}

With the increase of axial ratio of the heat exchange tube on the windward side, it is generally conducive to the heat dissipation at the junction. The scouring of

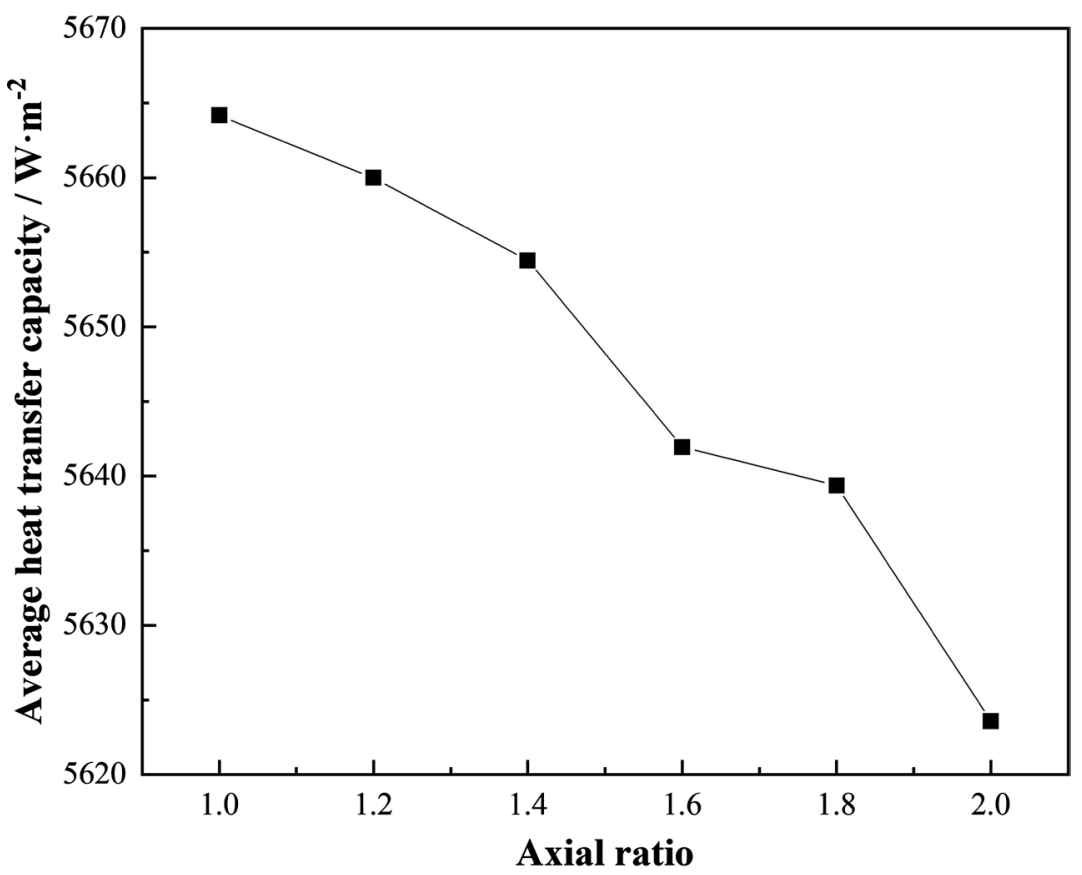

Figure 5. Effects of the change of the long and short axis ratio on the heat transfer capacity of the heat exchange tube surface. 
the windward side of the heat exchange tube by air is the main factor affecting the heat transfer on the windward side. The variation of the ratio of the long diameter and the short diameter causes the structural variation on the windward side, and the structural variations on the windward side directly affect the heat transfer capacity. The heat exchange capacity of the heat exchange tube surface slightly decreased as the increase of the ratio of the long and short axis of the heat exchange tube on the windward side, but the heat exchange area increases significantly, which raises the total heat exchange amount and improved the heat transfer performance of the radiator. When the ratio of the long axis and short axis increases from 1.0 to 2.0 , the average surface heat transfer capacity decreases from $5664.16 \mathrm{~W} / \mathrm{m}^{2}$ to $5623.57 \mathrm{~W} / \mathrm{m}^{2}$.

\section{Acknowledgements}

The authors wish to thank Shandong Provincial Natural Science Foundation, China (ZR2017LEE019) for their support of this study.

\section{Conflicts of Interest}

The authors declare no conflicts of interest regarding the publication of this paper.

\section{References}

[1] Mao, S.L., Cheng, C.R., Li X.C. and Michaelides, E.E. (2010) Thermal/Structural Analysis of Radiators for Heavy-Duty Trucks. Applied Thermal Engineering, 30, 1438-1446. https://doi.org/10.1016/j.applthermaleng.2010.03.003

[2] Wang, Y.M. (2016) Structure Optimization of Heat Tube and Heat Sink of Assembled Radiator for Vehicle. Ph.D. Thesis, Guizhou University, Guizhou.

[3] Petrik, M., Szepesi, G., Jármai, K. and Betti, B. (2017) Theoretical and Parametric Investigation of an Automobile Radiator. Vehicle and Automotive Engineering, 3, 27-37. https://doi.org/10.1007/978-3-319-51189-4_3

[4] Zheng, M.Q. (2016) Performance Analysis and Structure Optimization of Automobile Intercooler Based on CFD. Ph.D. Thesis, Guizhou University, Guizhou.

[5] Moon, M.A. and Kim, K.Y. (2014) Analysis and Optimization of Fan-Shaped Pin-Fin in a Rectangular Cooling Channel. International Journal of Heat and Mass Transfer, 72, 148-162. https://doi.org/10.1016/j.ijheatmasstransfer.2013.12.085

[6] Pan, A., Liu, X.N., Zheng, Z.H. and Li, W. (2020) Simulation and Optimization of the Structure of Radiator Fins with Venetian Finned Fins. Cryogenics \& Superconductivity, 48, 94-98.

[7] Shi, M., Du, J.W., Yu, X.L., Liao, Z.H. and Tan, Y.B. (2020) Orthogonal Numerical Simulation Optimition of Plain-Finned Heat Pipe Radiator. Electro-Mechanical Engineering, 36, 14-18.

[8] Pan, W.D. and Wu, J.H. (2007) The 3D Numerical Value Simulation of Automobile Radiator. Journal of Refrigeration, 26, 78-82. 